\title{
Fabrication of Ag and Ni Nanocatalyst with Enhanced Efficiency
}

\author{
Tajamal Hussain, ${ }^{1}$ Mira Tul Zubaida Butt, ${ }^{1}$ Mirza Nadeem Ahmed, \\ Muhammad Hamid Raza, ${ }^{1}$ Zahoor Hussain Farooqi, ${ }^{1}$ Khurram Shehzad, ${ }^{3}$ \\ Adnan Mujahid, ${ }^{1}$ and Rabia Batool ${ }^{1}$ \\ ${ }^{1}$ Institute of Chemistry, University of the Punjab, Lahore 54590, Pakistan \\ ${ }^{2}$ Institute of Chemistry, Government College University Faisalabad, Faisalabad 38030, Pakistan \\ ${ }^{3}$ Department of Information Science and Electronic Engineering, Zhejiang University, Hangzhou 310027, China \\ Correspondence should be addressed to Tajamal Hussain; tajamalhussain.chem@pu.edu.pk
}

Received 9 August 2015; Revised 21 September 2015; Accepted 29 September 2015

Academic Editor: Jean-Marie Nedelec

Copyright ( 2015 Tajamal Hussain et al. This is an open access article distributed under the Creative Commons Attribution License, which permits unrestricted use, distribution, and reproduction in any medium, provided the original work is properly cited.

Metal nanoparticles (NPs) have received significant attention in last decade because of their unique properties. In this work, two different metal NPs have been prepared and their catalytic activities are compared with conventional catalyst. In first case, Ag NPs were synthesized by chemical reduction method in ethanolic medium. Synthesized Ag NPs were characterized by scanning electron microscope (SEM) images which indicated an average particle size of Ag around $250 \mathrm{~nm}$. The catalytic activity of Ag NPs was investigated for the oxidative mineralization of methylene blue dye. Comparative studies suggested that Ag NPs possess enhanced catalytic activity compared to bulk Ag. In second approach, supported Ni NPs were fabricated using $\mathrm{Al}_{2} \mathrm{O}_{3}$ as supporting surface; that is, Ni NPs get adsorbed on $\mathrm{Al}_{2} \mathrm{O}_{3}$ through in situ reduction reaction. Oxidative degradation of methylene blue indicated that catalytic activity of supported $\mathrm{Ni} / \mathrm{Al}_{2} \mathrm{O}_{3}$ is about five times higher than simple $\mathrm{Ni}$ as catalyst.

\section{Introduction}

Development in the field of nanotechnology is gaining pace day by day because of its widespread applications. Scientists are successful in organizing the matter on such a small size [1-4]. Exploitation of the nanomaterials due to their many fundamental characteristics in widespread applications is the main reason behind ongoing research efforts in this field. Nanomaterials are considered to be the building blocks of next generations of catalysis, sensing, electronics, and medicines [4-7]. It is expected that conversion of bulk material into nanosize improves the functionality of the material. On the same line, considerable stimulation in the catalytic activity of the conventional catalysts takes place on converting them into nanoscale [8]. Catalysis is a surface phenomenon and size of catalyst somewhere in between bulk and molecular size materials leads to the highest efficiency of the catalyst since the highest percentage of the atoms is present on this level. Past decade has witnessed the trend of using nanosize catalyst rather than bulk or conventional catalyst $[9,10]$. Catalyst of smaller scale, for example, nanocatalyst, has widespread application in various fields [11, 12]. Metal NPs have their own identity among the nanosize catalysts since their properties are very much size dependent. Metal NPs are specific and have good efficiency when they are used as catalyst for various chemical reactions. Nowadays research on metal nanocatalysts revolves around three fields like their assembling or synthesis, structural and surface study, and applications. In present work, we reported the synthesis of Ag NPs in ethanolic medium while such synthesis takes place in aqueous medium along some capping agent, normally. Here ethanol played role of medium as well as capping agent. Morphological study of the synthesized NPs has been carried out using SEM. Catalytic activity of the prepared Ag NPs was investigated for oxidative mineralization reaction of methylene blue. Owing to the importance of $\mathrm{Ni}$ as catalyst [13], supported nanocatalyst of $\mathrm{Ni}$ on alumina is prepared by adsorption during the in situ reduction reaction. 
In addition, these synthesized supported Ni NPs were subjected to investigate the catalytic activity for oxidation of methylene blue as for Ag NPs.

\section{Experimental}

2.1. Chemical and Equipment. All the chemicals, $\mathrm{H}_{2} \mathrm{O}_{2}$ (Merck), $\mathrm{Al}_{2} \mathrm{O}_{3}(\mathrm{BDH}), \mathrm{NiNO}_{3}$ (Sigma Aldrich), $\mathrm{AgNO}_{3}$ (Sigma Aldrich), $\mathrm{NaBH}_{4}$ (Sigma Aldrich), and methylene blue (Sigma Aldrich), were used as purchased. UV-Vis spectrophotometer (PG Instruments Ltd., T90+) was used for observing oxidation of methylene blue. SEM (Hitachi, S4700) was used to study the surface morphology of NPs. Atomic absorption spectrometer (Perkin Elmer, AAnalyst 700) was used to calculate the amount of $\mathrm{Ni}$ adsorbed.

2.2. Preparation of Ag NPs by Reduction Method. Solution of $\mathrm{AgNO}_{3}$ was prepared by dissolving $0.34 \mathrm{~g}$ of $\mathrm{AgNO}_{3}$ in $24 \mathrm{~mL}$ ethanol. On the other hand, $100 \mathrm{~mL}$ of $0.1 \mathrm{M} \mathrm{NaBH}_{4}$ solution was prepared. Excess of $\mathrm{NaBH}_{4}$ solution was added for the better growth of $\mathrm{Ag} \mathrm{NPs}$. Solution of $\mathrm{NaBH}_{4}$ was transferred in a burette and added dropwise into $\mathrm{AgNO}_{3}$ solution with strong stirring. Upon adding few drops of sodium borohydride solution, grayish solid particles immediately appeared. After the complete addition of $\mathrm{NaBH}_{4}$ solution, reaction mixture was vigorously stirred for another 10 mins. Vacuum filtration was used to separate the Ag NPs. The solution was filtered with the help of polytetrafluoroethylene (PTFE) membrane. Finally, the synthesized NPs were dried in oven at $50^{\circ} \mathrm{C}$ for $1 \mathrm{hr}$ in the absence of air and stored in inert environment.

2.3. Oxidative Mineralization of Methylene Blue with Hydrogen Peroxide Catalyzed by Ag NPs. In order to investigate the catalytic activity of formed Ag NPs, methylene blue was reacted with $\mathrm{H}_{2} \mathrm{O}_{2}$ in the presence of prepared $\mathrm{Ag}$ NPs and absence of Ag NPs. Same amount of bulk Ag is added when kinetics was studied in the absence of Ag NPs. $2.5 \times 10^{-5} \mathrm{M}$ solution of methylene blue was prepared by dissolving $0.8 \mathrm{mg}$ of dye in $100 \mathrm{~mL}$ of deionized water. $0.7 \mathrm{M}$ $\mathrm{H}_{2} \mathrm{O}_{2}$ solution was prepared by adding $7 \mathrm{~mL}$ of $35 \%$ (w/w) solution of $\mathrm{H}_{2} \mathrm{O}_{2}$ in $100 \mathrm{~mL}$ of deionized water. In this particular kinetic experiment, $20 \mathrm{~mL}$ of standard methylene blue solution was mixed with $15 \mathrm{~mL}$ of $\mathrm{H}_{2} \mathrm{O}_{2}$ in the presence of Ag NPs and bulk Ag. Progress of the reaction was studied using spectrophotometer by noting absorbance of methylene blue at $\lambda_{\max }$ that is $665 \mathrm{~nm}$ [14].

2.4. Synthesis of Ni Nanocatalyst Supported on Alumina. $\mathrm{NiNO}_{3}$ solution of $0.1 \mathrm{M}$ was prepared by dissolving $1.21 \mathrm{~g}$ of $\mathrm{NiNO}_{3}$ in $100 \mathrm{~mL}$ of distilled water. After the addition of $5 \mathrm{~g}$ of $\mathrm{Al}_{2} \mathrm{O}_{3}$ in $\mathrm{NiNO}_{3}$ solution, excess amount of $0.1 \mathrm{M}$ solution of $\mathrm{NaBH}_{4}$ was added during the magnetic stirring. Magnetic stirring was continued for one hour at room temperature after the addition of $\mathrm{NaBH}_{4}$. Afterward, the $\mathrm{Al}_{2} \mathrm{O}_{3}$ containing $\mathrm{Ni}$ NPs adsorbed on its surface was filtered and washed with distilled water. Adsorbed Ni NPs on alumina was kept in oven at $60^{\circ} \mathrm{C}$ for $1 \mathrm{hr}$ in order to dry. To calculate the amount of adsorbed $\mathrm{Ni}$ on alumina, remaining amount of $\mathrm{Ni}$

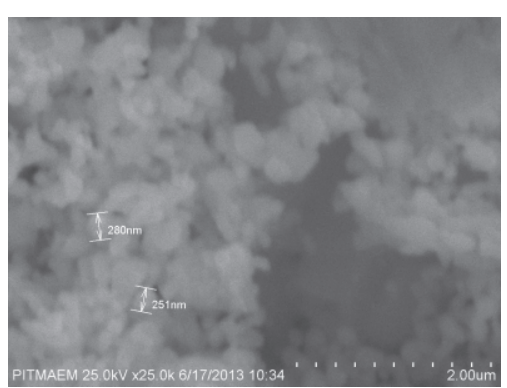

FIGURE 1: SEM image of silver NPs synthesized by chemical reduction method.

present in the filtrate was estimated using atomic absorption spectrometer.

2.5. Oxidative Mineralization of Methylene Blue with Hydrogen Peroxide Catalyzed by $\mathrm{Ni}_{\mathrm{A}} \mathrm{l}_{2} \mathrm{O}_{3}$. To investigate the catalytic efficiency of the synthesized supported Ni nanocatalyst for oxidation of methylene blue, $\mathrm{H}_{2} \mathrm{O}_{2}$ was used for oxidation of methylene blue in the presence of supported Ni NPs and simple Ni. Reaction kinetics for oxidation of methylene blue was examined exactly in the same way as for Ag NPs using spectrophotometer.

\section{Results and Discussion}

3.1. Preparation of Ag NPs by Reduction Method. Ag NPs were synthesized by chemical reduction method in ethanol using sodium borohydride as reducing agent. In the reaction mixture, sodium borohydride immediately converted Ag ions into nanosized Ag with zero oxidation state. These synthesized Ag NPs were stabilized by ethanol. Synthesis of such NPs was commonly accomplished in aqueous medium which often leads to agglomeration of NPs resulting in larger size of final material. However, using ethanol [15] solved this problem as it works as both solvent and capping agent leading to improved dispersion of NPs. The synthesized Ag NPs were characterized by SEM image as shown in Figure 1. This image suggests that Ag NPs were formed in the form of spherical granules showing suitable uniformity in size and shape. The average particle size of Ag NPs was between 250 and $280 \mathrm{~nm}$.

3.2. Oxidative Mineralization of Methylene Blue Catalyzed by $\mathrm{Ag} \mathrm{NPs}$. In order to investigate the catalytic activity of $\mathrm{Ag}$ NPs, they were used in oxidative mineralization of methylene blue dye. The decrease in absorbance of methylene blue as function of time was observed by UV-Vis spectrophotometer that shows the progress of catalysis reaction. The change in absorbance is directly related to the oxidation of methylene blue thus giving information about catalytic activity of $\mathrm{Ag}$ NPs. Figure 2 presents the change in absorbance of methylene blue as function of time. It was observed that concentration of the dye tends to decrease with the time. This decrease in the concentration was more pronounced when Ag NPs were used as catalyst compared with bulk Ag. This change in the catalytic activity of $\mathrm{Ag}$ is mainly due to its reduced size. 


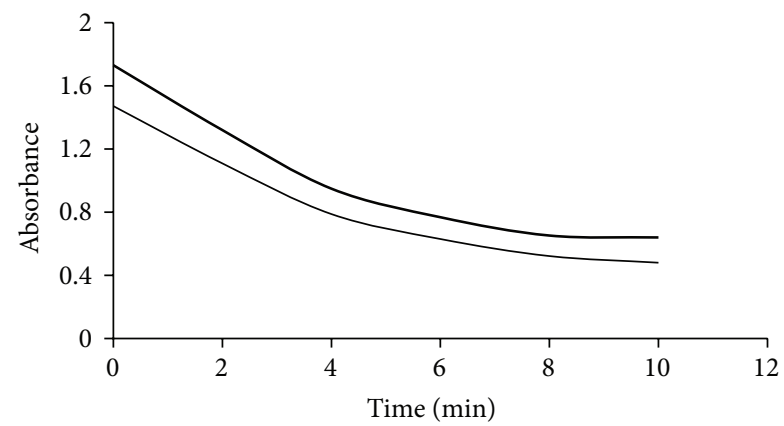

FIGURE 2: Time resolved profile of oxidation reaction of methylene blue in the presence (thin line) and absence (thick line) of Ag NPs as catalyst.

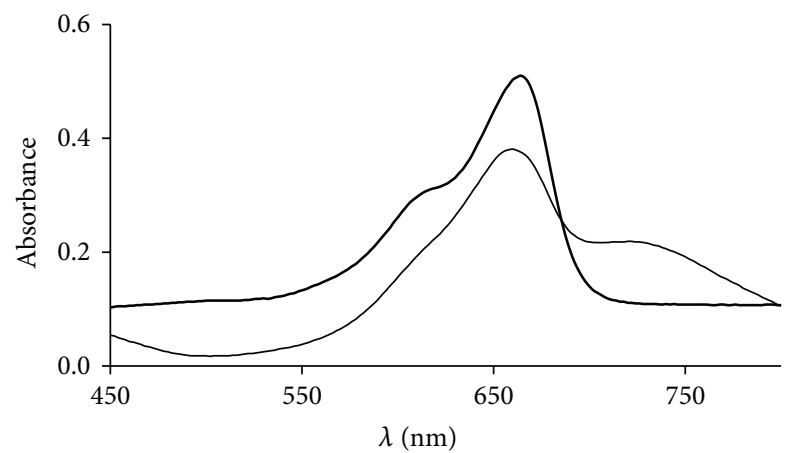

FIGURE 3: UV-Vis spectra of methylene blue during oxidation by $\mathrm{H}_{2} \mathrm{O}_{2}$ in the presence of $\mathrm{Ni}$ (thick) and $\mathrm{Ni} / \mathrm{Al}_{2} \mathrm{O}_{3}$ (thin).

Improved surface to volume ratio offers more interaction sites which ultimately improves catalytic activity.

\subsection{Oxidative Mineralization of Methylene Blue Catalyzed by} $\mathrm{Ni}$ and $\mathrm{Ni} / \mathrm{Al}_{2} \mathrm{O}_{3}$. The catalytic activity of Ni NPs adsorbed on alumina was also studied for oxidative degradation of methylene blue. UV-Vis spectra of methylene blue recorded during the oxidation by $\mathrm{H}_{2} \mathrm{O}_{2}$ in the presence of simple $\mathrm{Ni}$ and alumina supported Ni NPs are given in Figure 3. It can be observed that in case of alumina supported $\mathrm{Ni}$ NPs the decrease in absorbance of methylene blue was comparatively higher than nonsupported $\mathrm{Ni}$. The absorbance of the methylene blue dye in the presence of the nanocatalyst as a function of time at the maximum absorbance value, that is, $665 \mathrm{~nm}$, is shown in Figure 4. It is noted that sharp change in the absorbance of dye was observed when supported $\mathrm{Ni}$ NPs were used compared with simple Ni. Like Ag NPs, size of Ni NPs also came into play during the catalysis. The trend of decreasing absorbance with time is not same for both curves. For time intervals, between 5 and 9 mins and 15 and 20 mins there is relatively low decrease in absorbance for alumna supported Ni. This could be explained as first methylene blue adsorbed on the surface of alumina and then went through oxidation phase. It can be seen that the change in catalytic activity is more pronounced with the passage of time suggesting that methylene blue undergoes rapid degradation after getting adsorbed on alumina support.

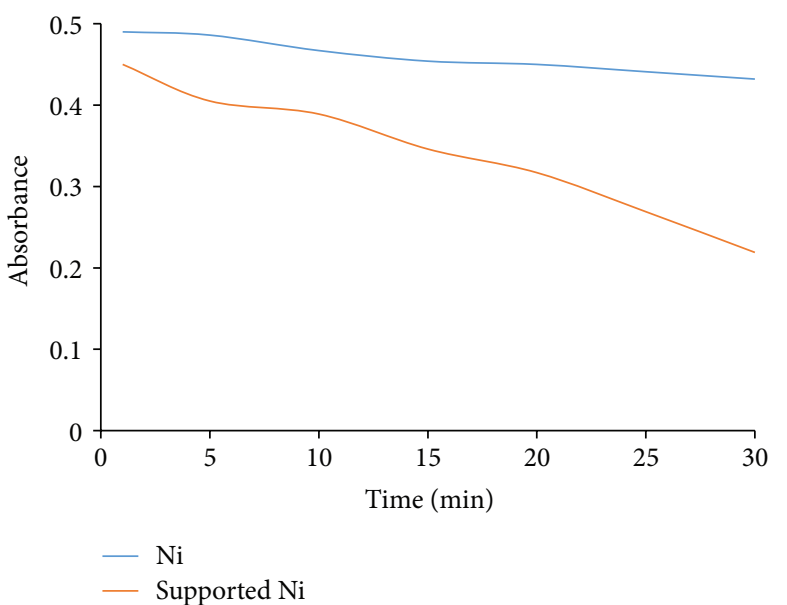

Figure 4: Absorbance of methylene blue during oxidation by $\mathrm{H}_{2} \mathrm{O}_{2}$ in the presence of $\mathrm{Ni}$, catalyst, as function of time.

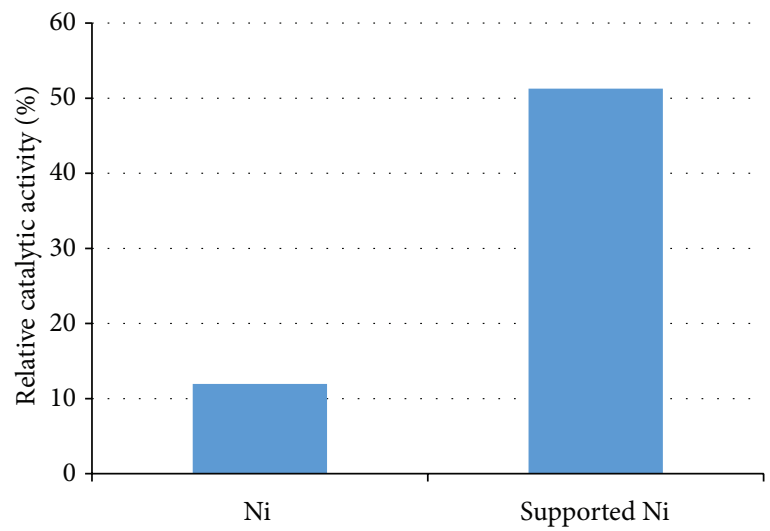

FIGURE 5: Relative percentage catalytic activity of simple $\mathrm{Ni}$ and supported Ni NPs for oxidation of methylene blue 30 minutes after the start of reaction.

Figure 5 is showing the relative percentage catalytic activity of simple $\mathrm{Ni}$ and supported Ni NPs for oxidation of methylene blue after 30-minute time. This relative percentage catalytic activity is calculated through change in concentration of dye after first 30 minutes of oxidation reaction. This shows that reaction was five times more accelerated when supported Ni NPs were used compared with simple Ni. This indicates that along the size of the catalyst, availability of the catalyst is also an important factor that accelerates the oxidation reaction.

\section{Conclusion}

Ag NPs have been successfully synthesized using ethanol instead of aqueous medium; furthermore, following this synthetic strategy, use of capping agent could be avoided. Moreover, ethanol can be considered as good solvent for having Ag NPs of narrow size range as shown by SEM analysis. Concerning catalytic activity, use of Ag NPs for oxidation reaction of methylene blue is favorable as it results improved efficiency when compared to bulk Ag. Supported 
Ni catalyst, results obtained from catalytic activity study were also pretty promising. Supported metal NPs as catalyst can be synthesized by one step in situ reduction reaction. Alumina supported Ni NPs have shown significantly high catalytic activity for oxidative degradation of methylene blue. This suggests that along the high surface to volume ratio the availability of catalyst interface plays an important part in catalysis reactions.

\section{Conflict of Interests}

The authors declare that there is no conflict of interests in this paper.

\section{Acknowledgment}

Tajamal Hussain would like to acknowledge the University of the Punjab, Lahore, Pakistan, for providing research grant to accomplish this project.

\section{References}

[1] A. A. Ayi, C. A. Anyama, and V. Khare, "On the synthesis of molybdenum nanoparticles under reducing conditions in ionic liquids," Journal of Materials, vol. 2015, Article ID 372716, 7 pages, 2015

[2] R. Jamil, T. Hussain, S. Saliha, and M. S. Ansari, "Magnetic field effects on the microstructural variation of electrodeposited nickel film," Journal of Materials Science and Engineering A, vol. 1, no. 4, pp. 481-487, 2011.

[3] Z.-M. Dang, K. Shehzad, J.-W. Zha et al., "Complementary percolation characteristics of carbon fillers based electrically percolative thermoplastic elastomer composites," Composites Science and Technology, vol. 72, no. 1, pp. 28-35, 2011.

[4] T. Hussain, A. T. Shah, K. Shehzad et al., "Formation of self-ordered porous anodized alumina template for growing tungsten trioxide nanowires," International Nano Letters, vol. 5, no. 1, pp. 37-41, 2015.

[5] A. Mujahid, A. Najeeb, A. I. Khan et al., "Tailoring of imprinted titinia nanoparticles for purines recognition," Journal of Materials, vol. 2015, Article ID 903543, 5 pages, 2015.

[6] K. Shehzad, M. N. Ahmad, T. Hussain et al., "Influence of carbon nanotube dimensions on the percolation characteristics of carbon nanotube/polymer composites," Journal of Applied Physics, vol. 116, no. 6, Article ID 064908, 2014.

[7] S. Peretz and O. Regev, "Carbon nanotubes as nanocarriers in medicine," Current Opinion in Colloid \& Interface Science, vol. 17, no. 6, pp. 360-368, 2012.

[8] T. Hussain, A. Mujahid, M. Ashraf, F. Tufail, H. Raza, and K. Shehzad, "Modified metal catalysts with enhanced surface area and their activity," NUST Journal of Engineering Sciences, vol. 7, no. 1, pp. 38-41, 2015.

[9] A. T. Shah, M. I. Din, U. Farooq et al., "Fabrication of nickel nanoparticles modified electrode by reverse microemulsion method and its application in electrolytic oxidation of ethanol," Colloids and Surfaces A: Physicochemical and Engineering Aspects, vol. 405, pp. 19-21, 2012.

[10] L. Shang, T. Bian, B. Zhang et al., "Graphene-supported ultrafine metal nanoparticles encapsulated by mesoporous silica: robust catalysts for oxidation and reduction reactions,"
Angewandte Chemie International Edition, vol. 53, no. 1, pp. 250254, 2014.

[11] W.-J. Ong, M. M. Gui, S.-P. Chai, and A. R. Mohamed, "Direct growth of carbon nanotubes on $\mathrm{Ni} / \mathrm{TiO}_{2}$ as next generation catalysts for photoreduction of $\mathrm{CO}_{2}$ to methane by water under visible light irradiation," RSC Advances, vol. 3, no. 14, pp. 45054509, 2013.

[12] C. T. Wirth, B. C. Bayer, A. D. Gamalski et al., "The phase of iron catalyst nanoparticles during carbon nanotube growth," Chemistry of Materials, vol. 24, no. 24, pp. 4633-4640, 2012.

[13] S. Chen, J. Duan, J. Ran, M. Jaroniec, and S. Z. Qiao, "N-doped graphene film-confined nickel nanoparticles as a highly efficient three-dimensional oxygen evolution electrocatalyst," Energy \& Environmental Science, vol. 6, no. 12, pp. 3693-3699, 2013.

[14] N. Kannan and M. M. Sundaram, "Kinetics and mechanism of removal of methylene blue by adsorption on various carbons-a comparative study," Dyes and Pigments, vol. 51, no. 1, pp. 25-40, 2001.

[15] Q. Wang, S. Snyder, J. Kim, and H. Choi, "Aqueous ethanol modified nanoscale zerovalent iron in bromated reduction: synthesis, charectrization and reactivity," Environmental Science and Technology, vol. 43, no. 9, pp. 3292-3299, 2009. 

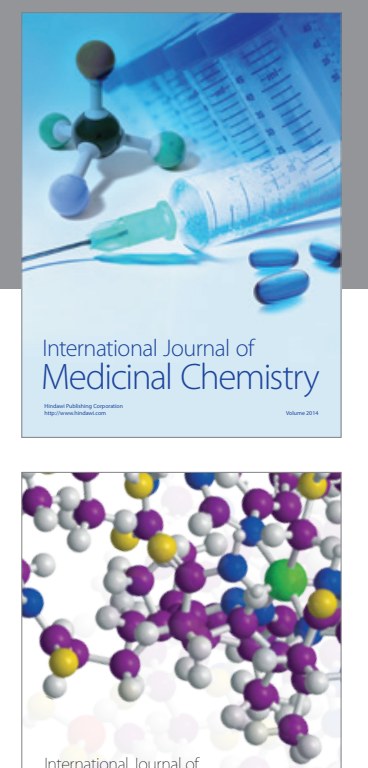

\section{Carbohydrate} Chemistry

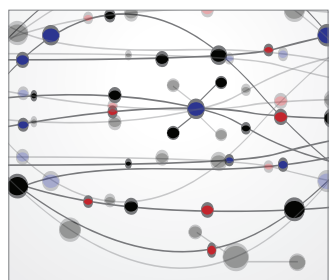

The Scientific World Journal
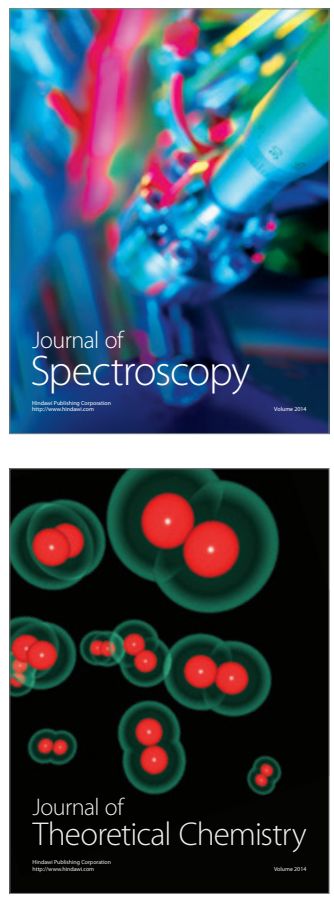
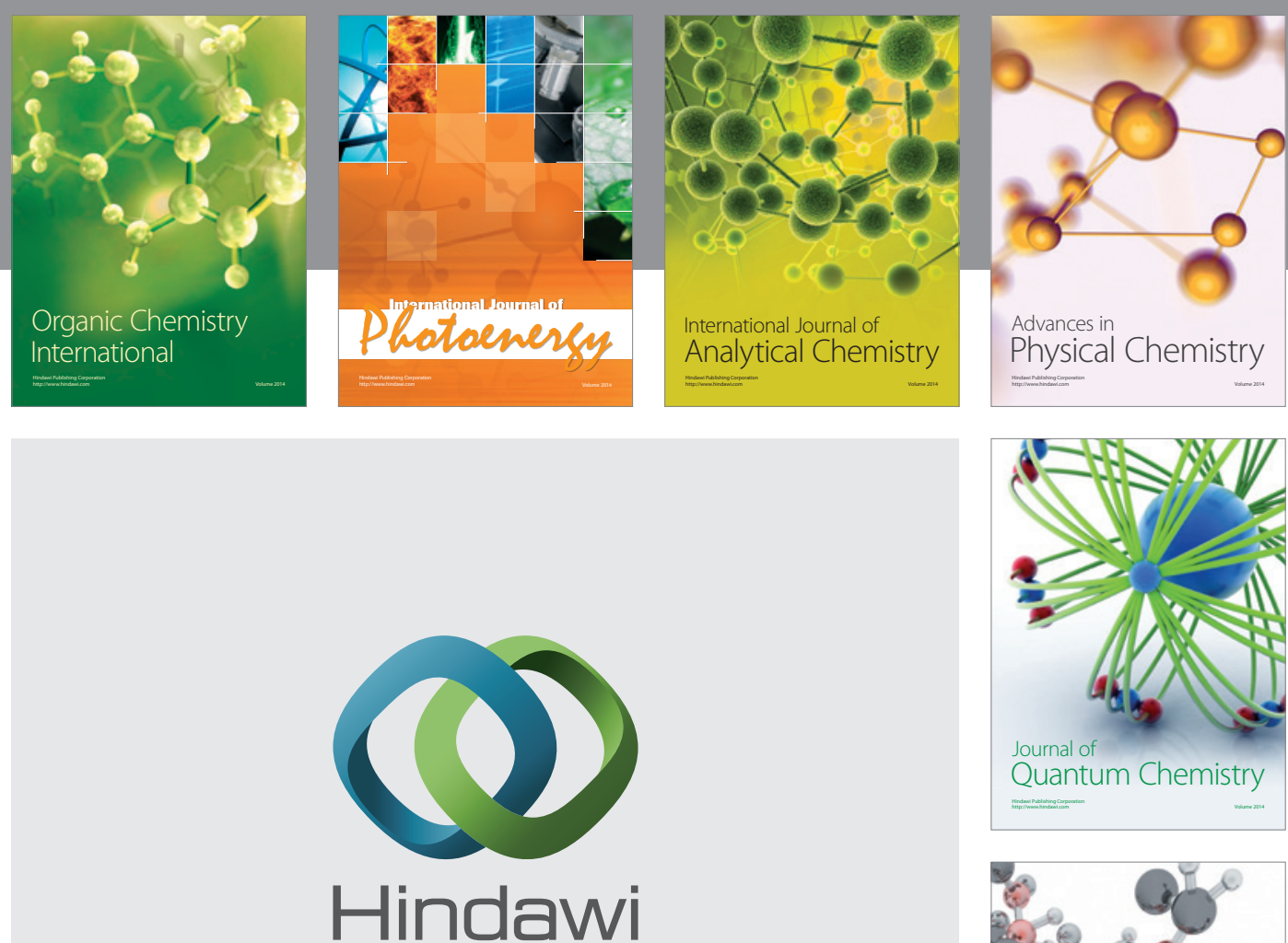

Submit your manuscripts at

http://www.hindawi.com

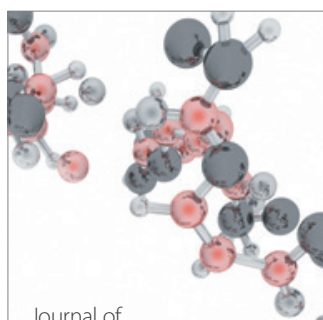

Analytical Methods

in Chemistry

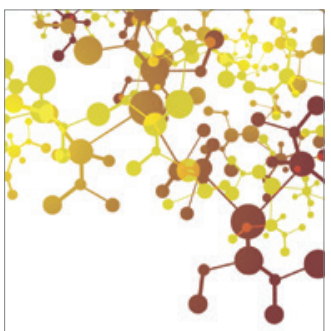

Journal of

Applied Chemistry

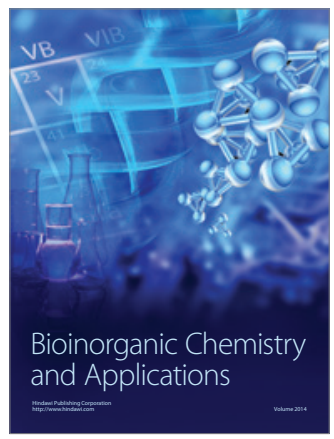

Inorganic Chemistry
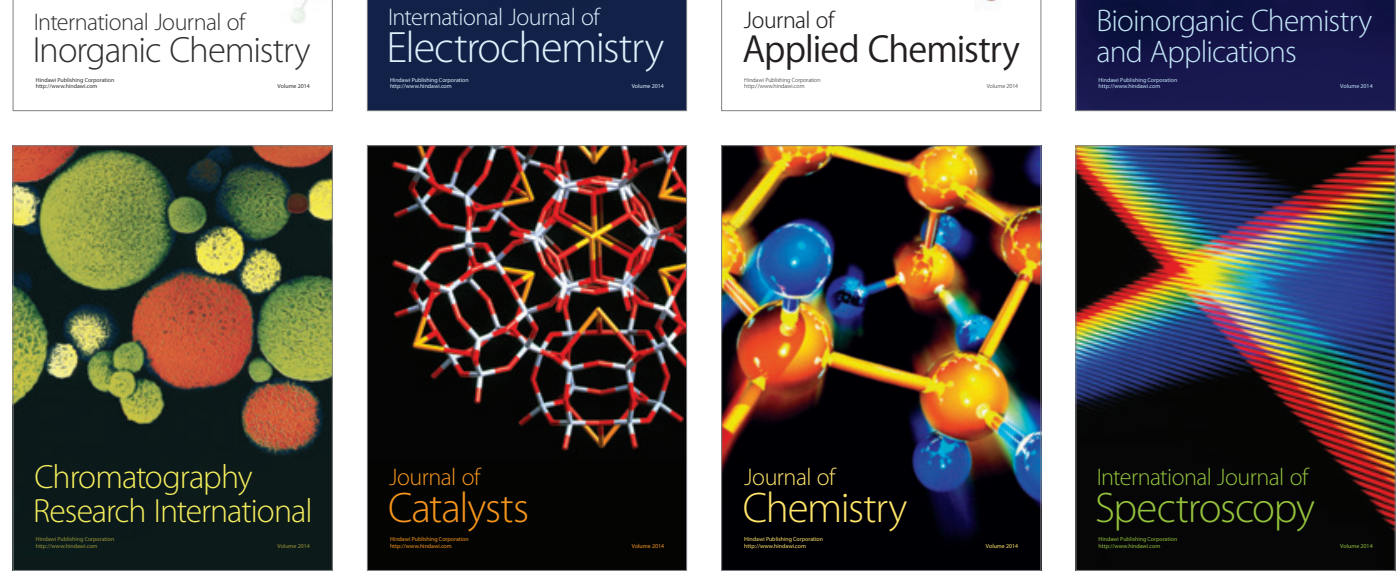